University of Nebraska - Lincoln

DigitalCommons@University of Nebraska - Lincoln

\title{
Influence of van der Waals forces on increasing the strength and toughness in dynamic fracture of nanofibre networks: a peridynamic approach
}

Florin Bobaru Ph.D.

University of Nebraska at Lincoln, fbobaru2@unl.edu

Follow this and additional works at: https://digitalcommons.unl.edu/engineeringmechanicsfacpub

Part of the Applied Mechanics Commons, Biomaterials Commons, Computational Engineering Commons, Engineering Mechanics Commons, Mechanics of Materials Commons, Molecular, Cellular, and Tissue Engineering Commons, and the Polymer and Organic Materials Commons

Bobaru, Florin Ph.D., "Influence of van der Waals forces on increasing the strength and toughness in dynamic fracture of nanofibre networks: a peridynamic approach" (2007). Faculty Publications from the Department of Engineering Mechanics. 70.

https://digitalcommons.unl.edu/engineeringmechanicsfacpub/70

This Article is brought to you for free and open access by the Mechanical \& Materials Engineering, Department of at DigitalCommons@University of Nebraska - Lincoln. It has been accepted for inclusion in Faculty Publications from the Department of Engineering Mechanics by an authorized administrator of DigitalCommons@University of Nebraska - Lincoln. 


\title{
Influence of van der Waals forces on increasing the strength and toughness in dynamic fracture of nanofibre networks: a peridynamic approach*
}

\author{
F Bobaru ${ }^{1}$ \\ ${ }^{1}$ Department of Engineering Mechanics, University of Nebraska-Lincoln, Lincoln, \\ NE 68588-0526, USA
}

Received 7 December 2006, in final form 21 March 2007

Published 7 June 2007

Online at stacks.iop.org/MSMSE/15/397

\begin{abstract}
The peridynamic method is used here to analyse the effect of van der Waals forces on the mechanical behaviour and strength and toughness properties of three-dimensional nanofibre networks under imposed stretch deformation. The peridynamic formulation allows for a natural inclusion of long-range forces (such as van der Waals forces) by considering all interactions as 'long-range'. We use van der Waals interactions only between different fibres and do not need to model individual atoms. Fracture is introduced at the microstructural (peridynamic bond) level for the microelastic type bonds, while van der Waals bonds can reform at any time. We conduct statistical studies to determine a certain volume element for which the network of randomly oriented fibres becomes quasi-isotropic and insensitive to statistical variations. This qualitative study shows that the presence of van der Waals interactions and of heterogeneities (sacrificial bonds) in the strength of the bonds at the crosslinks between fibres can help in increasing the strength and toughness of the nanofibre network. Two main mechanisms appear to control the deformation of nanofibre networks: fibre reorientation (caused by deformation and breakage) and fibre accretion (due to van der Waals interaction). Similarities to the observed toughness of polymer adhesive in the abalone shell composition are explained.
\end{abstract}

(Some figures in this article are in colour only in the electronic version)

\section{Introduction}

\section{1. modelling of fibre networks: importance and literature review}

Recent advances in manufacturing nanofibre networks and membranes from continuous nanofibres via electrospinning (e.g. [1,2]) offer numerous opportunities for applications of

* The author would like to dedicate this work to the 60th anniversary of Professor Subrata Mukherjee. 
these new material systems to special filters, tissue engineering, drug delivery systems, reinforcements in composite materials, etc [2-4]. The most popular manufacturing method for producing continuous fibres, electrospinning, has been modeled and analysed (see, e.g. [5,6]) with the goal of improving the reliability and control of the process. In the applications area the efforts have been mainly experimental. The mechanical performance of nanofibre networks in terms of their strength and damage and fracture properties has only recently received attention (see, e.g. [7, 8]). The ability to tailor the properties of nanofibre networks and membranes requires good understanding of the mechanical properties of the nanofibre networks, and systematic modelling and analysis of these material systems may provide the necessary information to optimize their structure (nanofibre orientation, diameter, density of contacts and fused contacts, etc) for specific applications.

Methods for analysing the mechanical behaviour of stochastic fibre networks have been proposed in, for example, [9-11]. Fused porous networks are analysed in [12] where special attention is paid to the geometry and effective mechanical properties of the interconnects. Percolation in wavy fibre networks is analysed in [13] where it was observed that the percolation threshold increased with an increase in the fibre degree of curliness. These results are for finite length fibres.

An effective-medium model is introduced in [14] for the elasticity of two-dimensional random fibre networks. These networks are constructed from short fibres such as those found in paper. The exact statistics to describe the microscopic geometry of the network is used and it is shown that the tensile modulus can be expressed by a single-parameter function. This parameter depends on the network density and fibre dimensions, which relate the macroscopic modulus to the relative importance of axial and bending deformations of the fibres. An analytical and a numerical model are used in [15] to investigate the in-plane stiffness of fibre mats. A mat is modelled by randomly depositing thin linear-elastic fibres on top of each other under the influence of an external pressure. The fibres are assumed rigidly bonded at contacts. The stiffness is given as a function of only a few parameters: the degree of bonding, the dimensions of the fibres, the elastic constants of the fibre material and the density of fibres. A very similar micromechanics model is given in [16] for the elasticity of planar fibre networks made from short fibres. The rods are still bonded rigidly at contacts in [16] and under external in-plane loading. An effective-medium constitutive relation for the fibre network is developed. Calculations are performed to analyse the effects of the fibre aspect ratio and the fibre concentration on the effective stiffness of the planar fibre network.

An analysis of the influence of filament misalignment on the toughening of lightweight ballistic fabrics is presented in [17]. The analysis, based on a continuum effective-medium theory, refers to woven fabrics and it is observed that introducing misalignment is desirable since this will macroscopically toughen the fabric. The inhomogeneity of individual filament ruptures is considered to be the main mechanism through which the material absorbs more energy.

Numerical models for simulating the deformation and fracture of finite length polyethylene (PE) fibres at the nanoscale are proposed in [18]. An ordered network of PE molecules is represented using a two-dimensional lattice with the nodes being linked by elastic rods that carry normal and shear forces to represent the intrachain covalent bonds and the interchain van der Waals forces. No crosslinking is used. Eyring kinetic theory of fracture is used to model the lattice response and the equilibrium is reached with the Monte Carlo method. It is assumed that the van der Waals bonds for which the shear strain in equilibrium is below $10 \%$ can reform. The influence of the strain rate and the temperature on the tensile behaviour and the deformation micromechanism are studied.

van der Waals forces are always present and play a role in (see, e.g. [19]) adhesion ( [20]), surface tension, properties of this films, strength of solids and structure of macromolecules 
(such as proteins and polymers). They are nonadditive (see chapter 6 in [19]) in the sense that the force between any two molecules is affected by the presence of other molecules nearby. The difference between the van der Waals energy between two molecules in a medium and that calculated from the consideration of pair interactions alone can be as large as $\pm 20 \%$. This difference is particularly large for interactions between large particles and surfaces in a medium (see chapter 11 in [19]). In the present study we ignore the existence of a medium (air) engulfing the nanofibre network; thus, the additivity assumption for the van der Waals forces is reasonable.

Membrane-like networks of electrospun polymer continuous fibres of nanometre scale diameters have been recently produced in the laboratory (e.g. Li and Xia [1], Dzenis [2], Ramakrishna et al [3]). It is of importance to analyse the mechanical behaviour of such networks built from randomly oriented fibres or from aligned fibres.

A method that has shown great potential for the modelling of complex material systems is the peridynamic method developed by Silling [21]. Branching cracks and fragmentation in dynamic fracture has been a very challenging problem to simulate numerically. In high-aspect ratio structures, such as thin shells and membranes and fibrous materials, the computational difficulties faced by classical approaches are multiplied by numerical ill-conditioning. The peridynamic method, used in this paper, reformulates the classical continuum mechanics equations by replacing the spatial derivatives with an integral of force densities over a region around the material point of interest. This leads to a nonlocal method that may be thought of as a continuum version of molecular dynamics simulation. Any two material points are connected by an, possibly nonlinear, elastic spring (bond) with a critical stretch parameter. Certain similarities with other types of nonlocal methods exist (e.g. Eringen and Edelen [22], Eringen [23], Kunin [24]), as well as important differences. In particular, the peridynamic method does not require averaging of some measure of infinitesimal strain over a neighbourhood of the material particle (see [21, 25-27]).

Nonlocal methods have been studied for several decades; however, they have not yet been widely adopted in computational simulations of materials and structures. A review of the status of nonlocal modelling of materials damage (such as microcracking, void formation and softening frictional slip) is given in [28]. One of the problems facing nonlocal models is that a large effort is required to develop the material models employed by these methods. To reduce this burden, alternatives that can improve on the capabilities of local continuum models have also been proposed, such as lattice models for modelling damage in concrete (see, e.g. [29-32]. The peridynamic method offers new promise in connecting existing material models developed in the classical continuum mechanics literature with nonlocal continuum models that can approximate the dispersion of short elastic waves, for example. The direct connection between peridynamics and classical elasticity is explained in $[21,25,33]$ and nonlinear elasticity in [26]. Work is currently under way to allow peridynamic formulations (PFs) to use existing constitutive models in plasticity, thermoelasticity, etc.

The main advantages of the peridynamic method compared with the existing computational methods are the following.

- Treatment of fracture without special techniques; the crack tip profile is cusp-like even when linear-elastic bonds are used [21].

- Meshfree discretization allows capturing damage and fracture naturally. Cracks initiate spontaneously when and where they are energetically favourable [26,27].

- The underlying equations naturally allow for the inclusion of long-range forces, such as van der Waals forces, that are important at small size scales [26].

- Versatile treatment of high-aspect ratio structures and complex heterogeneous materials [34]. 


\subsection{Our contributions}

We present examples of dynamic fracture and damage for high-aspect ratio structures such as nanofibre networks. We model a new class of materials (nanofibre networks) under imposed displacement boundary conditions. We simulate the mechanical behaviour of 3D fibre networks under dynamic fracture and damage until total failure of the structure. We analyse possible strengthening and toughening mechanisms of the nanofibre network when van der Waals forces are present and when sacrificial bonds exist between individual fibres in the crosslink bonds.

The aim is to employ model reduction in which, instead of modelling individual atoms, we use discretization points that represent an average response of hundreds or thousands of atoms by

- using an elastic (harmonic) potential along each fibre to model the elastic forces in the fibres,

- using the elastic potential to compute forces between nodes on two different fibres at the fused crosslinks and

- using the van der Waals potential to model the van der Waals interaction between any two different fibres.

A convergence study is performed for the reduced model to determine the appropriate node density that can replicate the overall effect of van der Waals forces with sufficient accuracy.

The diameter of the nanofibres considered here is $\sim 1.2 \mathrm{~nm}$ and is determined by the parameters used in the van der Waals interaction: when two fibres are closer than $\sim 1.2 \mathrm{~nm}$ the van der Waals force becomes repulsive. The parameters used in this qualitative study are not meant to provide a quantitative description of the studied phenomena. Continuous nanofibres with diameters as low as $20 \mathrm{~nm}$ and discontinuous fibres with diameters of $5 \mathrm{~nm}$ have been produced through electrospinning [35]. However, continuous nanofibres with diameters smaller than about $50 \mathrm{~nm}$ cannot yet be produced uniformly and repeatedly for most materials systems [2].

\subsection{Paper organization}

The paper is organized as follows: in section 2 we introduce a new algorithm for generating nanofibre networks inside the given bounded regions. The peridynamic model used here is presented in section 3 where, after a brief review, we show how van der Waals and elastic potentials are combined, and we perform a convergence study for the effect of the van der Waals interaction between two different nanofibres. In section 4 we describe the generation of a specific network of randomly oriented nanofibres which is then subjected to biaxial strain in order to obtain an isotropic-response 'representative volume element' (RVE). Further numerical simulations are provided in section 5 to distinguish between two main deformation mechanisms and assess the influence of sacrificial bonds on the overall strength and toughness of the nanofibre network. We conclude in section 6.

\section{Generating nanofibre networks}

As seen in figures 4 and 5 in [1] or figure 7.28 in [3], for example, the nanofibres produced by electrospinning have some waviness and multiple contacts, some of them possibly fused. To create an equivalent computational model for a fibrous network with a membrane-like structure, such as those produced by electrospinning deposition of nanofibres on a flat surface, we 
developed a numerical algorithm that generates continuous fibres inside a bounding box. As the height of the bounding box becomes smaller, we approach the membrane-like construction of fibres. The procedure listed in algorithm 1 (see also figure 1) is used to generate families of directional strings with certain randomness built in along each string.

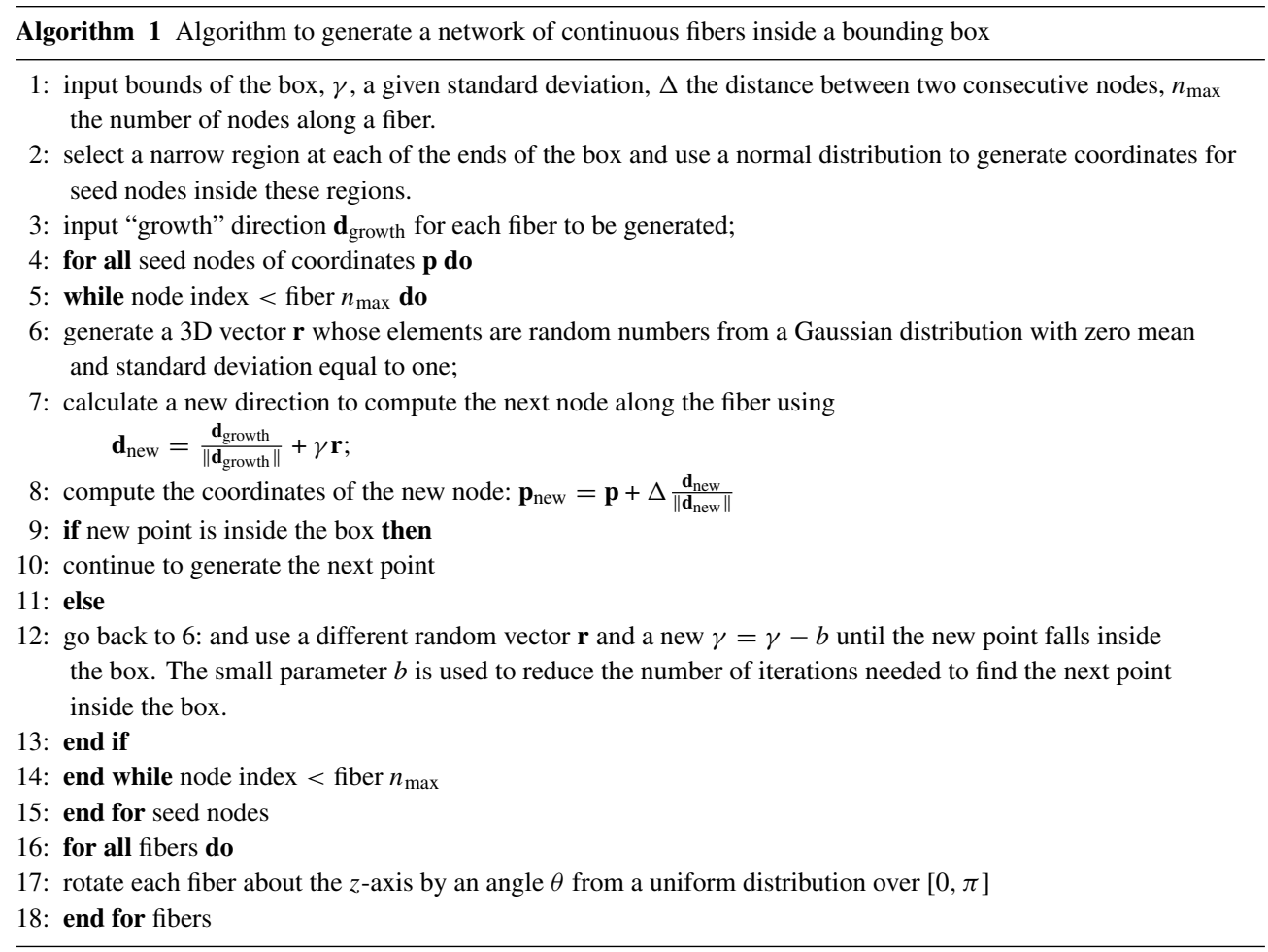

This process of generating fibres networks can be generalized to include a locationdependent standard deviation $\gamma=\gamma(\tau, m)$, with $\tau$ being, for example, the arclength along each fibre and $m$ being the fibre number (or the fibre family number). The waviness of each fibre could thus be correlated to match with experimentally observed shapes of real fibres in various types of networks. This is not pursued here since most electrospinning processes generate fibres that are mostly straight, with some small variability. The case of constant $\gamma=0.3$ is shown in figure 1 where two families of fibres are generated inside a parallelepipedic box along two orthogonal directions. Other types of bounding boxes can be easily used (see Bobaru and Silling [7]).

\section{The peridynamic model}

The peridynamic theory [21] is based on a reformulation of the classical continuum mechanics equations in which the divergence of the stress term is replaced by an integral of the force acting at a material point due to interactions with the rest of the material. This leads to a nonlocal theory that is fundamentally different from earlier nonlocal elastic models (e.g. [22,23]) since in the PF no spatial derivatives appear. The consequences of eliminating spatial derivatives are critical for fracture and phase changes problems, because, unlike the classical theory, the mathematical structure of the peridynamic theory is unaffected by the emergence of these discontinuities. 

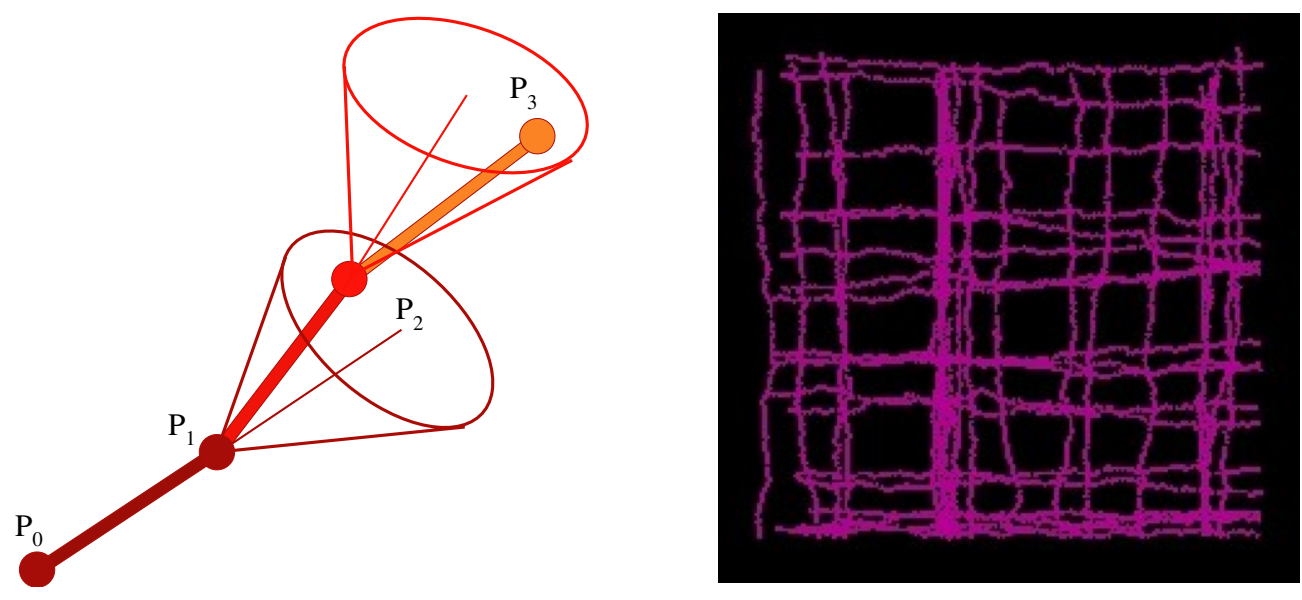

Figure 1. The new node $P_{2}$ is generated along a direction which stays inside a cone whose height is along the $P_{0} P_{1}$ direction. The angle of the cone is related to the magnitude of the input standard deviation $\gamma$. This process is repeated for all nodes in a fibre. The picture on the right shows the resulting fibre network when 20 fibres are generated along the horizontal direction and 20 along the vertical direction, inside a thin parallelepiped. A constant value of $\gamma=0.3$ was used for all fibres.

In this paper we briefly review the PF and describe several examples of dynamic fracture and damage of materials/structures with very large aspect ratios: thin membranes and networks of nanofibres. As suggested by the notion of a continuum version of molecular dynamics, in the $\mathrm{PF}$ the acceleration of any particle $\mathrm{P}$ in the reference configuration at time $t$ is found from the equation of motion:

$$
\rho \ddot{\boldsymbol{u}}(\boldsymbol{p}, t)=\int_{H_{\boldsymbol{p}}} \boldsymbol{f}_{\boldsymbol{p} \boldsymbol{q}}(\boldsymbol{q}-\boldsymbol{p}, \boldsymbol{u}(\boldsymbol{q}, t)-\boldsymbol{u}(\boldsymbol{p}, t)) \mathrm{d} \boldsymbol{q}+\boldsymbol{b}(\boldsymbol{p}, t),
$$

where $\boldsymbol{p}$ is the position vector for particle $\mathrm{P}$ (see figure 2 ) in the reference configuration, $H_{\boldsymbol{p}}$ is a neighbourhood of the particle $\mathrm{P}$, while $\mathrm{Q}$ is a generic particle, with position vector $\boldsymbol{q}$, inside this neighbourhood. The vector $\boldsymbol{u}(\boldsymbol{p}, t)$ is the displacement vector field of particles at time $t$ (the current configuration), $\boldsymbol{b}(\boldsymbol{p}, t)$ is a prescribed body force density field at $\mathrm{P}$ at time $t, \rho$ is the mass density in the reference configuration and $f_{p q}$ is a pairwise force function whose value is the force vector (per unit volume squared) that particle $\mathrm{P}$ exerts on particle $\mathrm{Q}$. We denote the relative position vector of these two particles in the reference configuration by $\boldsymbol{r}=\boldsymbol{q}-\boldsymbol{p}$. The relative displacement vector is $\boldsymbol{u}_{\boldsymbol{r}}=\boldsymbol{u}(\boldsymbol{q}, t)-\boldsymbol{u}(\boldsymbol{p}, t)=\boldsymbol{u}_{\boldsymbol{q}}-\boldsymbol{u}_{\boldsymbol{p}}$. Using these definitions, the vector $\tilde{\boldsymbol{r}}=\tilde{\boldsymbol{q}}-\tilde{\boldsymbol{p}}=\boldsymbol{r}+\boldsymbol{u}_{\boldsymbol{r}}$ represents the current relative position vector between the two particles. The direct physical interaction (which occurs through, so far, unspecified means) between the particles at $\boldsymbol{p}$ and $\boldsymbol{q}$ is called a peridynamic bond or simply a bond. The bonds extend over a finite distance around a particle; this is a fundamental difference between the PF and the classical continuum mechanics, which is based on the idea of contact forces (interactions between particles that are in direct contact with each other). It is convenient to assume that for a given material there is a positive number $h$, called the horizon, such that if $\|\boldsymbol{r}\|>h$ then $\boldsymbol{f}_{\boldsymbol{p} \boldsymbol{q}}\left(\boldsymbol{r}, \boldsymbol{u}_{\boldsymbol{r}}\right)=\mathbf{0}$. By definition (see [21]) a material is said to be microelastic if the pairwise force function is derivable from a scalar micropotential $w$ :

$$
\boldsymbol{f}_{\boldsymbol{p q}}\left(\boldsymbol{r}, \boldsymbol{u}_{\boldsymbol{r}}\right)=\frac{\partial w_{p q}\left(\boldsymbol{r}, \boldsymbol{u}_{\boldsymbol{r}}\right)}{\partial \boldsymbol{u}_{\boldsymbol{r}}} .
$$

The micropotential $w$ is the strain energy in a single bond and has dimensions of energy per unit volume squared. The energy per unit volume in the body at a given point (i.e. the 


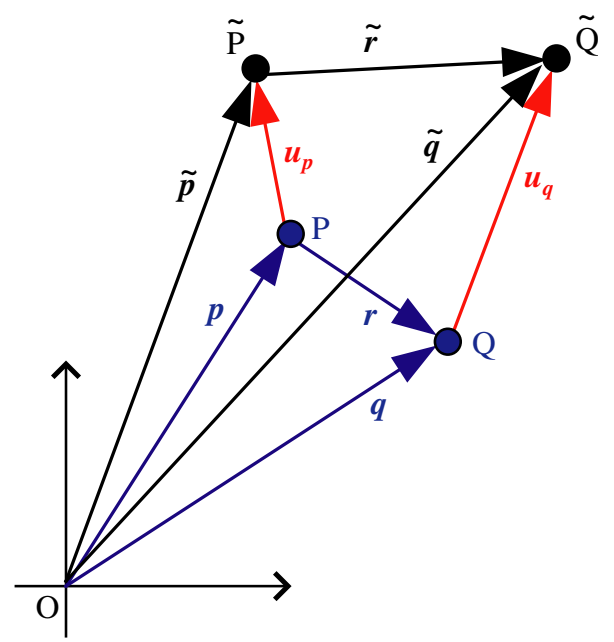

Figure 2. The material particles (peridynamic nodes) $\mathrm{P}$ and $\mathrm{Q}$ and their corresponding position vectors in the reference configuration and the current configuration denoted by the tilde.

local strain energy density) is therefore found by integrating the strain energy in all bonds between the given point and surrounding material points within the horizon (see, e.g. [21,26]). If a body is composed of a microelastic material, work done on it by external forces is stored in a recoverable form in the same way as in the classical theory of elasticity. It can be shown [21] that the micropotential depends on the relative displacement vector $\boldsymbol{u}_{\boldsymbol{r}}$ only through the scalar distance between the deformed points: $w_{p q}=w_{p q}(\boldsymbol{r},\|\tilde{\boldsymbol{r}}\|)$. For isotropic materials, this relationship can be further written as $w_{p q}=w_{p q}(\|\boldsymbol{r}\|,\|\tilde{\boldsymbol{r}}\|)$.

\subsection{Microelastic potential}

Bonds with linear-elastic behaviour (the force in a bond is proportional with the strain in the bond) can be modelled using a quadratic micropotential. Let us first define the bond strain as

$$
s_{p q}(\|\boldsymbol{r}\|,\|\tilde{\boldsymbol{r}}\|)=\frac{\|\tilde{\boldsymbol{r}}\|-\|\boldsymbol{r}\|}{\|\boldsymbol{r}\|} .
$$

Note that the bond strain (3) for the bond between material particles with position vectors $\boldsymbol{p}$ and $\boldsymbol{q}$ in the reference configuration is not the classical infinitesimal strain. The bond strain is a 'finite' strain which does not involve spatial differentiation. This is one major difference between the $\mathrm{PF}$ and the classical formulations with important consequences for modelling fracture. In the limit of the horizon converging to zero, the peridynamic theory for microelastic materials converges to the classical, local elasticity. The microelastic force can then be written as

$$
\boldsymbol{f}_{\boldsymbol{p} \boldsymbol{q}}(\boldsymbol{r}, \tilde{\boldsymbol{r}})=\frac{\partial w_{p q}}{\partial s_{p q}} \frac{\tilde{\boldsymbol{r}}}{\|\tilde{\boldsymbol{r}}\|} g(\|\boldsymbol{r}\|) .
$$

The function $g$ defines whether bonds with different reference lengths have different elastic responses. In practice, $g$ has little effect except with regard to deformations that occur on a length-scale smaller than the horizon (an analysis of this is provided in Xie's thesis [36]). For example, the wave dispersion properties of the material at small wavelengths are affected by terms of this type (see [21]). The size of the horizon and the particular shape of the function $g$ may, in principle, be correlated with experimental results for various materials. On the other 
hand, the horizon does not have to be necessarily viewed as a material length-scale (see, for example, [37]).

For linear microelastic materials the potential for homogeneous isotropic bodies is given by $w_{p q}=\frac{1}{2} C s_{p q}^{2}$, with $C$ a positive constant. This leads to the linear microelastic force

$$
\boldsymbol{f}_{\boldsymbol{p} q}\left(\boldsymbol{r}, \boldsymbol{u}_{\boldsymbol{r}}\right)=C s_{p q} \frac{\tilde{\boldsymbol{r}}}{\|\tilde{\boldsymbol{r}}\|} g(\|\boldsymbol{r}\|) .
$$

The constant $C$ can be related to the bulk modulus $\kappa$ in classical elasticity by equating the elastic strain energy at a point in the classical and the PFs (see [27]): $C=18 \kappa / \pi h^{4}$ in three dimensions. Similar relationships can be obtained in one and two dimensions. In order to simulate the propagation of a wave in a classical elastic material with peridynamics, certain practical values for the node density and the horizon size relative to the wavelength of a propagating perturbation need to be used. Results along these lines are found in [36-38].

\subsection{Nanoscale effects}

For nanostructured materials, such as nanofibre networks, long-range forces, such as van der Waals forces, may play an important role in the mechanics of deformation of such structures. The PF allows the inclusion of such forces in a natural way. In addition to the micropotential given above, we consider a Lennard-Jones-(LJ) type potential that acts between any two material points, but only if they belong to different fibres. To avoid having to model each individual atom as in molecular dynamics simulations, we choose to use the elastic interactions (the microelastic peridynamic forces) between material points along an individual fibre and augment that with van der Waals forces acting between fibres. Points of contact between two fibres may or may not have microelastic bonds. van der Waals interactions will always be present between nodes on two different fibres as long as the nodes are in the action range of this force. The range of action of the long-range forces (van der Waals) does not have to be the same as the horizon of the peridynamic forces. The total potential we use in this case is

$$
\begin{aligned}
& w_{\boldsymbol{p} \boldsymbol{q}}(\|\boldsymbol{r}\|,\|\tilde{\boldsymbol{r}}\|)=w_{\boldsymbol{p} \boldsymbol{q}}^{\mathrm{el}}(\|\boldsymbol{r}\|,\|\tilde{\boldsymbol{r}}\|)+w_{\boldsymbol{p q}}^{\mathrm{LJ}}(\|\tilde{\boldsymbol{r}}\|), \\
& \text { with } w_{\boldsymbol{p} \boldsymbol{L}}^{\boldsymbol{L J}}(\|\tilde{\boldsymbol{r}}\|)=\alpha\left(\frac{a}{\|\tilde{\boldsymbol{r}}\|}\right)^{12}-\beta\left(\frac{a}{\|\tilde{\boldsymbol{r}}\|}\right)^{6},
\end{aligned}
$$

where the $\mathrm{LJ}$ potential $w^{\mathrm{LJ}}$ depends only on the relative distance in the current configuration, $\|\tilde{\boldsymbol{r}}\|=\left\|\boldsymbol{r}+\boldsymbol{u}_{\boldsymbol{r}}\right\|$. Note that the LJ potential generates a force density per unit volume. The equation in (1) is modified to include an integral of this force density over a different horizon than that used in the structural elastic interaction.

We ignore the presence of air. Due to the highly porous structure of our nanofibre network, the ' $1 / 6$ ' pair potential between the nanofibres seems reasonable. Note that for two parallel chain molecules (see [19]), the interaction energy calculated based on the ' $1 / 6$ ' atomic pair potential is proportional to the length of the chains and inversely proportional to $\sigma^{2}$ and $d^{5}$ where $\sigma$ is the molecular diameter and $d$ is the separation distance between the two chains. In our study we maintain the ' $1 / 6$ ' power law between material particles for several reasons: (a) our fibres are not aligned initially and (b) we model a generic polymer-like material; therefore no molecular diameter is specified. The convergence study described in section 3.4 provides further motivation for this selection.

\subsection{Damage and fracture in the $P F$}

Damage may be incorporated into a peridynamic constitutive model by allowing the bonds for solid interactions to break irreversibly (e.g. Silling and Bobaru [26], Silling and Askari 
[27]). The simplest assumption is that this breakage occurs when a bond is extended beyond some predetermined critical bond deformed length. Since the breakage is irreversible, time and position must now be included as arguments in the interparticle force, for example: $\hat{f}\left(\boldsymbol{r}, \boldsymbol{u}_{\boldsymbol{r}}, t\right)=f\left(\boldsymbol{r}, \boldsymbol{u}_{\boldsymbol{r}}\right) \mu_{\boldsymbol{p} \boldsymbol{q}}\left(s_{\boldsymbol{p} \boldsymbol{q}}, t\right)$, where $\mu$ is a history-dependent function defined by

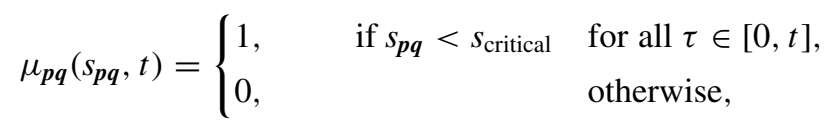

with $s_{\text {critical }}$ being the critical value of bond strain for breakage to occur. Recall that any particle in the continuum has an infinite number of bonds connecting it to other particles. During deformation, some of these bonds may break as determined by (7), and breakage of the bonds occurs independently among different bond lengths and orientations for a given particle. In practice, bond breakage in a peridynamic body usually evolves in such a way as to form two-dimensional surfaces. These surfaces correspond to cracks; their growth is the way fracture propagation occurs spontaneously in the peridynamic theory. The initiation and growth process occurs without reference to any supplemental kinetic relation that controls the crack growth on the basis of a stress intensity factor or a similar quantity. In this sense, fracture modelling in the peridynamic theory is 'autonomous' and represents a fundamental difference between the present theory and the techniques of traditional fracture mechanics. Convergence for crack growth in a peridynamic material is shown in Silling and Askari [27]. The van der Waals forces in our study are not subject to damage: they form and reform as dictated by the separation distance between peridynamic nodes.

\subsection{Peridynamic numerical model for nanofibre networks}

Membrane-like networks of electrospun polymer continuous fibres of nanometre scale diameters have been recently produced in the laboratory (e.g. [1,2]). It is of importance to analyse the mechanical behaviour of such networks built from randomly oriented fibres or from aligned fibres. In the examples given below we use a linear model for peridynamic bond elasticity with a critical bond strain $s_{\text {critical }}$ of $30 \%$ (at which point the bond breaks) and maximum compression of $10 \%$ (at which point contact repulsive forces prevent the interpenetration of material). The micromodulus is chosen to correspond to Young's macro modulus of $1.5 \mathrm{GPa}$ (see section 3.1). Measurements of the mechanical properties for individual electrospun nylon nanofibres have been obtained in [39] and they range between $0.5 \mathrm{GPa}$ to around $1 \mathrm{GPa}$ for fibres with diameters around $500 \mathrm{~nm}$. The fibres interact through peridynamic forces as well as through long-range forces (van der Waals forces) as described above. The latter are used only between nodes belonging to different fibres. These interactions influence the deformed shape of the network, which includes cellular patterns, as shown below. Fibres that are in contact with one another or interpenetrating are considered bonded through peridynamic (solid-like bonds). The constants in the LJ potential (7) we use in these examples are $\alpha=\beta=1$ and $a=1.1 \mathrm{~nm}$. This makes the van der Waals force repulsive for a separation distance less than about $s_{0}=1.235 \mathrm{~nm}$, while the peak (attractive) force is obtained around $s_{\max }=1.372 \mathrm{~nm}$. The force decreases to $10 \%$ of its peak value for a separation distance of $2 \mathrm{~nm}$. A convergence study is performed to determine the necessary spacing between the discretization nodes in fibres such that the total van der Waals force between parallel adjacent fibres is insensitive to the exact locations of the nodes. In figure 3 we show two such configurations for fibres of length $200 \mathrm{~nm}$. The distance between the two fibres is $d$. The spacing between nodes, $\Delta x$, varies from 0.2 to $2 \mathrm{~nm}$. For sufficiently dense nodes along each fibre, the vertical component of the total force exerted by one fibre on the other should be, roughly, the same. The plot in 

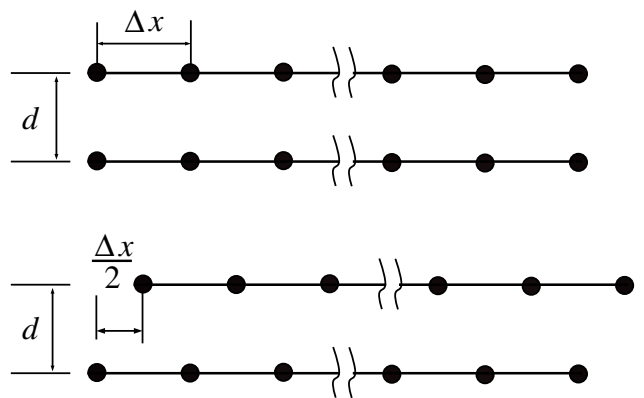

(a)

Distance between fibres $\mathrm{d}=1.5 \mathrm{~nm}$

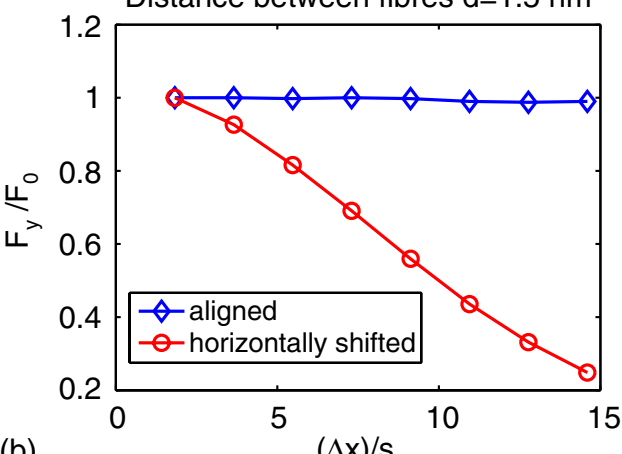

(b)

$(\Delta \mathrm{x}) / \mathrm{s}$

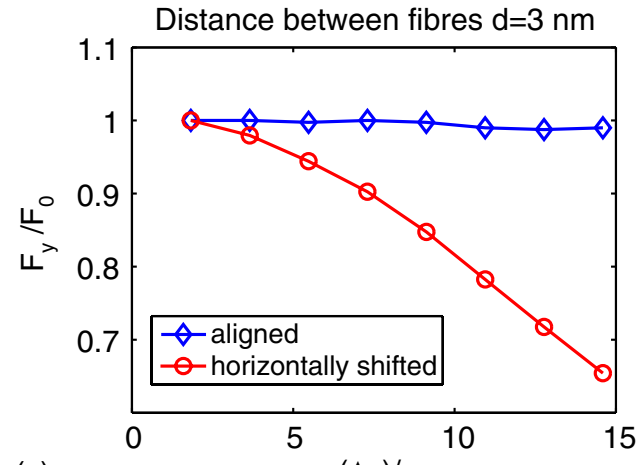

(c)

$(\Delta \mathrm{x}) / \mathrm{s}$

Figure 3. Convergence for the Van der Waals force between two $200 \mathrm{~nm}$ long fibres in terms of spacing between discretization nodes. (a) Two parallel fibres separated by a distance $d$ nm are placed in two configurations: aligned and shifted. (b) The vertical component of the total force (normalized) between fibres is measured as a function of the grid spacing (normalized) for the case when $d=1.5 \mathrm{~nm}$. (c) The vertical component of the total force (normalized) between fibres measured as a function of the grid spacing (normalized) for the case when $d=3 \mathrm{~nm}$.

figure 3 shows that at a distance of $1 \mathrm{~nm}$ between the nodes the dependence of the total force on the different, but practically equivalent, configurations becomes less than $10 \%$ when the fibres are $3 \mathrm{~nm}$ apart. The grid spacing data in figure 3 are normalized by the distance $s=s_{\max }-s_{0}$ for the abscissa, while the total vertical force $F_{y}$ is normalized by the limit value of this force as $\Delta x \rightarrow 0, F_{0}$. In the examples that follow we use this separation distance between the nodes along each fibre.

Bending stiffness is not directly considered in the present model. The bending stiffness of a nanofibre is indeed expected to be small (the bending stiffness scales with the cube of the fibre diameter). The nonlocal character of the peridynamic bonds induces a small amount of bending stiffness which is more pronounced in fibres that are not straight in their original configuration since on straightening or bending them some of the bonds will be stretched while others compressed. There are two ways in which a peridynamic model can directly model bending stiffness for the problem considered here: (a) introduce additional degrees of freedom (related to changes in angles between bonds) or (b) consider each fibre as a 3D cylinder and use the enhanced peridynamic model in [21]. The second option seems too costly from a computational point of view, since each individual fibre would require tens of thousands of discretization nodes. The first option is a subject of future work. 


\section{Determining the RVE}

We determine a RVE for a specific nanofibre network which should satisfy the following two requirements:

(i) the mechanical response is isotropic,

(ii) the total strain energy during dynamic deformation and fracture is the same for different stochastic realizations of the nanofibre network.

The size of the RVE will clearly depend on the architecture of the specific network, such as distribution and density of contacts, average length of free fibre, porosity, etc. In our case, the architecture is determined by the number of fibres generated, the size of the three-dimensional box in which the fibres are generated, the amount of waviness (controlled by parameter $\gamma$ in algorithm 1) along each fibre and the length between two consecutive nodes that define the fibre. The numerical results given below are obtained for a particular nanofibre network generated as described next. While all the obtained quantities apply to this particular network, the qualitative observations apply in general.

\subsection{Generating the network of randomly oriented fibres}

According to the algorithm presented in section 2, we select four end-regions of a parallelepiped of size $[-300: 300,-300: 300,-2.5: 2.5]$. In each end region we generate 25 random points (from a uniform distribution, respectively) that are starting nodes for directional fibres. The fibres from one end 'grow' along a direction such that they reach across to the opposite side of the box, with the corresponding direction chosen here as $\boldsymbol{i}, \boldsymbol{j},-\boldsymbol{i},-\boldsymbol{j}$ (the triplet $\{\boldsymbol{i}, \boldsymbol{j}, \boldsymbol{k}\}$ representing the unit cartesian vectors). Directional fibres are then formed with some randomness in their orientation (see algorithm 1). Along each fibre, the distance between two consecutive nodes is taken to be unity. A small standard deviation $\gamma=0.15$ (see figure 1) is used here motivated by the aspect of nanofibre produced by electrospinning. The 'directional' fibres generated in this way are shown in figure $4(a)$. We now rotate each fibre about the $z$-axis by a random angle taken from a uniform distribution. To avoid having fibres end inside the computational box we cut a sub-region of the original parallelepiped to the following limits: $[-l: l,-l: l,-2.5: 2.5]$, with $l \in[0,250]$. After rotation and cutting, a fibre network for a new $l_{\text {new }}=200$ is shown in figure $4(b)$.

Mechanically, if two fibres 'touch' or interpenetrate then elastic bonds are formed at the intersection nodes. These bonds have the same properties as the elastic bonds along the fibres. They are also nonlocal, in the sense that each node in a fused region of a fibre is connected to all the nodes, from its own fibre or other fibres, that fall inside its horizon. The effective diameter of a fibre is defined by the van der Waals interaction and in our case is around $1.4 \mathrm{~nm}$. Since the thickness of the bounding box is $5 \mathrm{~nm}$, and the horizon we used here is $3 \mathrm{~nm}$, many of the crossovers in figure $4(b)$ are not actually fused. However, even when elastic bonds between fibres do not exist, van der Waals forces are active between different fibres if they are close enough for these forces to act.

\subsection{Obtaining the $R V E$}

For the 100 fibres generated above, we find the RVE by applying biaxial extension to the network of size $[-l, l ;-l, l,-2.5,2.5]$ with $l \in[0,250]$. The displacement boundary conditions are applied over a length of $30 \mathrm{~nm}$ at each end of the network. This reduces the effective $x$ and $y$ dimensions of the network by $60 \mathrm{~nm}$ for every value of $l$ considered. 

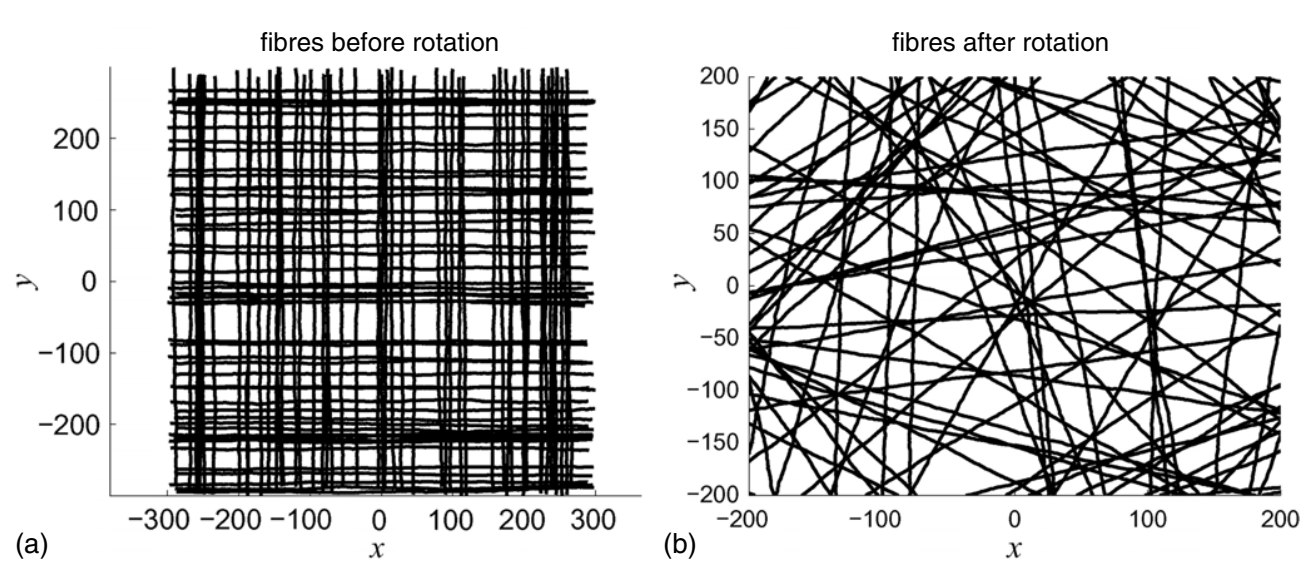

Figure 4. A three-dimensional fibre network with preferred growth directions (see algorithm 1 and figure 1) along the $x$ - and $y$-axes. The bounding box has limits as $[-300,300 ;-300,300 ;-2.5$, 2.5]. The randomly positioned 'directional' fibres $(a)$ and a volume cutout after rotating each fibre about the $z$-axis with a random angle selected from a uniform distribution $(b)$.

Constant velocities of magnitude $v_{0}=1 \mathrm{~nm} \mathrm{~ns}^{-1}$ are applied to each side of the network as a boundary condition to induce biaxial stretch. Initially, the points along the fibres have velocities proportional to the magnitude of their corresponding $x$ and $y$ coordinates. In other words, the initial horizontal velocity, for example, varies linearly along the $x$-direction from $-v_{0}$ at the left end of the network to $v_{0}$ at the right end of the network. This choice for the initial velocity is selected in order to avoid an initial shock wave propagation. The time-step used in computations satisfies the stability requirements given in [27]. The simulations are performed using the EMU code [40].

We measure the response (the total force that one half of the network exerts on the other half) along the directions perpendicular to the median planes parallel to the $x$ - and $y$-axes. We gradually increase the values of $l$ and note that for $l=200$ the responses for the two median cuts become very close to one another. The effective length of the network is $340 \mathrm{~nm}$ since the ends have imposed displacement conditions.

In figure 5 we show the undeformed and deformed configurations for one realization of the randomly orientated nanofibres. A sequence of snapshots is taken for network biaxial strains (in the $x$ - and $y$-directions) of about $10 \%$ (at $17 \mathrm{~ns}$ ), 26.5\% (at $45 \mathrm{~ns}$ ), 36.5\% (after $62 \mathrm{~ns}$ ) and $53 \%$ (after $90 \mathrm{~ns}$ ), respectively, for the $(b),(c),(d)$ and (e) plots. The magnitude of the force component perpendicular to the planes $x=0$ and $y=0$ is plotted versus time in figure 6 . Since the motion occurs with constant velocity, the time coordinate is proportional to the network strain, and therefore the area under the curve in figure 6 is proportional to the energy dissipated during the deformation. These results also give a measure of the nanofibre network ductility (strain to complete failure).

We perform the same numerical test for the case when the van der Waals forces are not included in the computation. The deformed shapes at the same amount of stretch as the ones corresponding to the graphs in figures $5(b)$ and $(c)$ are shown in figures $7(b)$ and (c). The time histories for the total force magnitude in the $x$-axis direction for the case without the van der Waals forces are shown in figure 8. Note the dramatic effect of the longrange forces acting at the nanoscale on the amount of energy dissipated. This observation is crucial in the different fracture behaviour observed in nanostructured biological materials (see, e.g. [41-43]). 

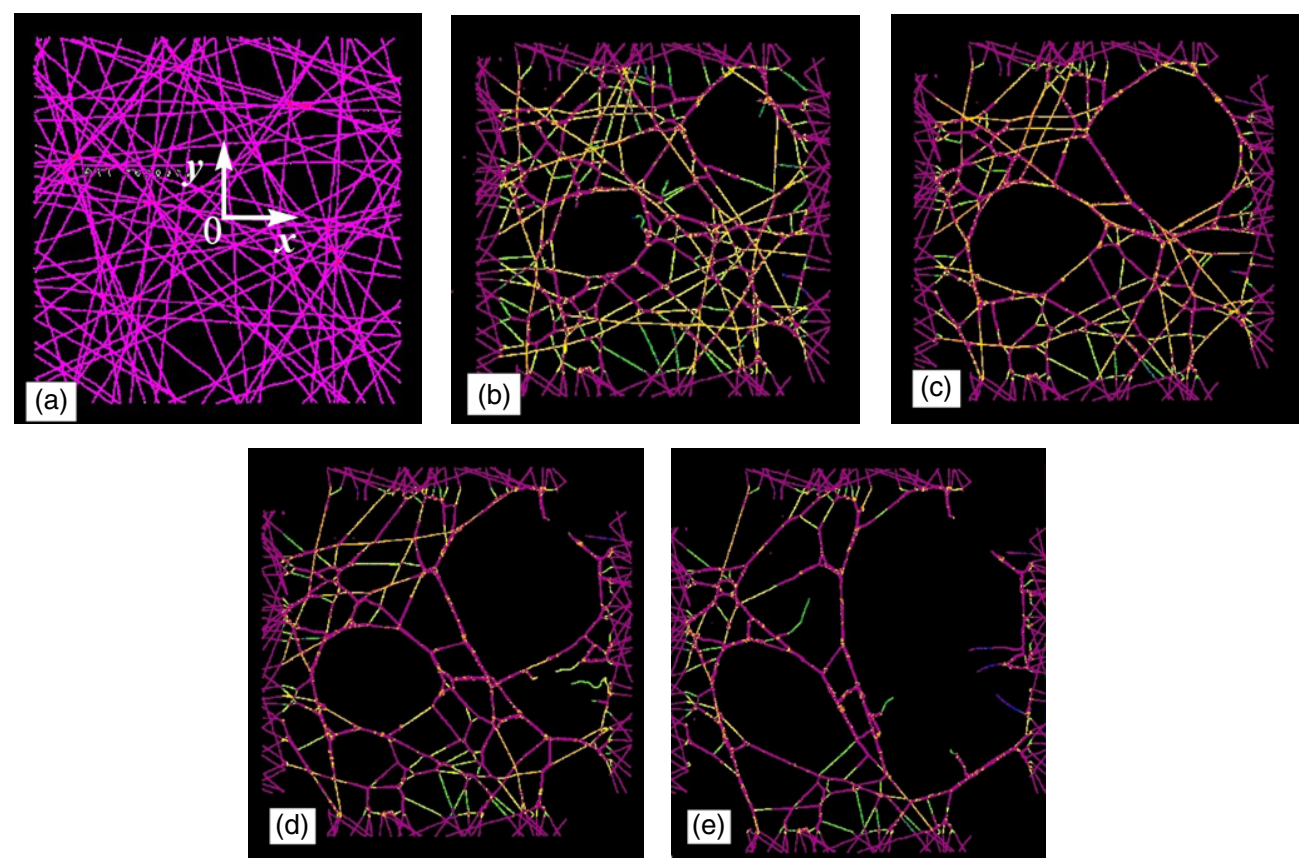

Figure 5. The undeformed $(a)$ and deformed configurations under progressively larger biaxial network strain of $10 \%(17 \mathrm{~ns})(b), 26.5 \%(45 \mathrm{~ns})(c), 36.5 \%(62 \mathrm{~ns})(d)$ and $53 \%(90 \mathrm{~ns})(e)$.
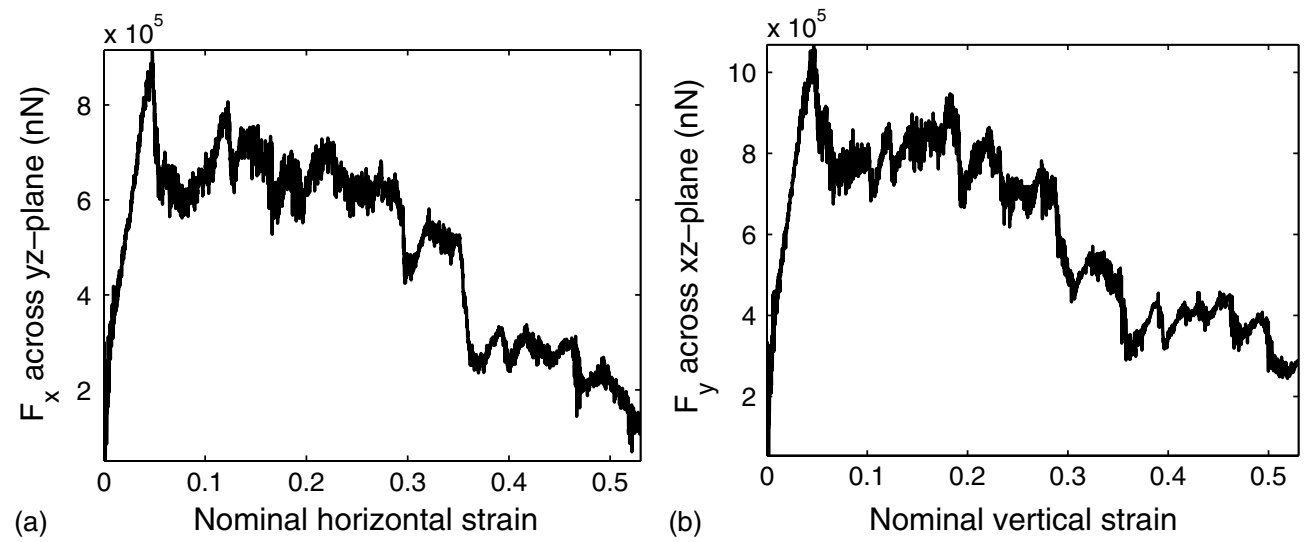

Figure 6. Magnitude of the total horizontal force component through the $y z$-plane $(a)$ and the total vertical force component through the $x z$-plane $(b)$ for biaxial stretching.

The additional test for determining the RVE is to see if, when generating different realizations of the fibre network, we obtain a similar response. We generate three different networks of 100 fibres as in figure $4(a)$ and select $l=200$ to perform the cutting after rotation (as in figure $4(b))$. The number of fibres which fall inside the box of $(-200<x<200,-200<$ $y<200,-2.5<z<2.5$ ) is 73,74 and 70 , respectively.

We monitor time histories for the total force across two planes: $x=0$ and $y=0$ (see figure $5(a)$ ). We estimate the total work consumed during the dynamic stretching process by 

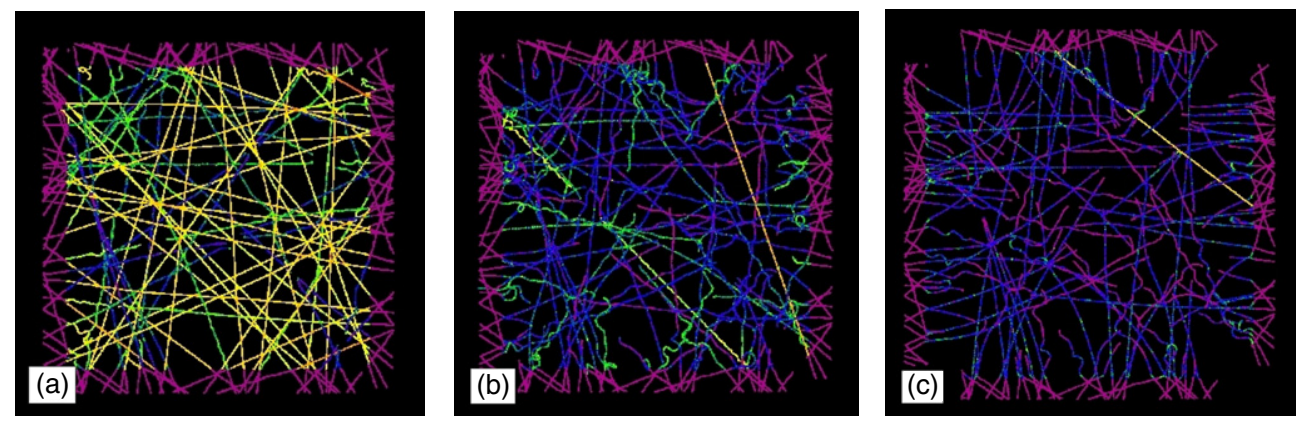

Figure 7. Deformed and damaged network when van der Waals forces are absent. The cases in $(b)$ and $(c)$ correspond to those in figures $5(b)$ and $(c)$, respectively ( $17 \mathrm{~ns}$ and $45 \mathrm{~ns})$. The plot in $(a)$ is for a stretch of $3.5 \%$ (after $6 \mathrm{~ns}$ ).
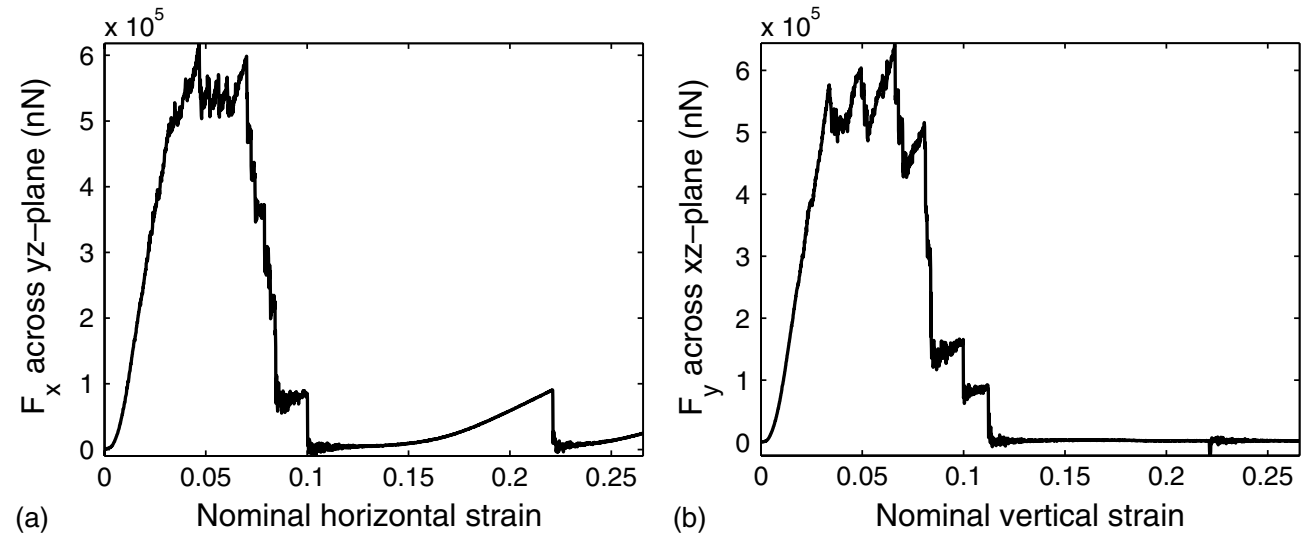

Figure 8. Magnitude of the total horizontal force component through the $y z$-plane $(a)$ and the total vertical force component through the $x z$-plane $(b)$ for biaxial stretching. The case without van der Waals forces.

using numerical integration (the trapezoidal rule) to evaluate the following integral:

$$
v_{0} \int_{0}^{t_{\text {final }}} F_{x} \mathrm{~d} t+v_{0} \int_{0}^{t_{\text {final }}} F_{y} \mathrm{~d} t
$$

where $F_{x}$ and $F_{y}$ are the $x$ and $y$ components of the total force vector through the cutting planes $x=0$ or $y=0$. The $z$ component is nonzero, but is insignificant compared with the other two components. Since constant velocity $v_{0}$ is used, the above equation can be considered as representative of the work done by stretching the fibre network $\left(\int \boldsymbol{F} \cdot \mathrm{d} s\right)$.

As shown in table 1, the dissipated energy (work done) at $t=45 \mathrm{~ns}$ varies by no more than $10 \%$ for the three cases and by no more than $3 \%$ at $t=90 \mathrm{~ns}$. The relative differences, per fibre, between the three cases are even smaller. Here, the simulation time of $90 \mathrm{~ns}$ is chosen as a representative time because the total force across the plane drops to less than $20 \%$ of its peak value. The strength left in the network is small compared with its maximum value. 
Table 1. Work (in nanoJoules) done (8) by the nanofibre network under biaxial stretching.

\begin{tabular}{lll}
\hline & $t_{\text {final }}=45 \mathrm{~ns}$ & $t_{\text {final }}=90 \mathrm{~ns}$ \\
\hline Case 1 (73 fibres) & $6.3364 \mathrm{e}+007$ & $9.8817 \mathrm{e}+007$ \\
Case 2 (74 fibres) & $7.0889 \mathrm{e}+007$ & $1.0669 \mathrm{e}+008$ \\
Case 3 (70 fibres) & $6.2163 \mathrm{e}+007$ & $9.7642 \mathrm{e}+007$ \\
\hline
\end{tabular}

\section{Numerical results}

\section{1. fibre reorientation and van der Waals-induced Poisson effect}

There are two noticeable factors that play a role in the mechanical deformation of nanofibre networks:

- reorientation (induced by the breakage of fibres and dynamic deformation) and

- accretion (generated by van der Waals forces, when present).

These phenomena are more clearly observed in the following numerical tests in which the top and bottom horizontal boundaries are free. This is the case of uniaxial extension. All conditions are similar to the biaxial strain case, except for the imposed velocity which is now increased to $3 \mathrm{~nm} \mathrm{~ns}^{-1}$. In figure 9 we show four different configurations at increasing amounts of horizontal strain. A Poisson-type effect is observed. This is induced by the accretion due to the van der Waals forces.

In the present model, strain-rate dependence of the deformation and failure of the nanofibre network is directly induced by the inertia term in the equations of motion. The effect is especially significant when adhesive forces are included, because when fibres have more time to reorient and adhere to each other the network will become stronger. We do not include any other types of strain rate dependence here. A detailed analysis of strain-rate dependence is a topic for future research.

The absence of the van der Waals interaction eliminates the Poisson effect observed before. We do not include the figures for this case because they are similar to the biaxial results in figure 7 , with the only difference being the boundary conditions imposed. The effect of the imposed deformation is fibre reorientation alone.

\subsection{Influence of van der Waals forces and sacrificial bonds}

We test three cases.

(A) Fully crosslinked, constant linkage strength. All crossovers are bonded (solid-like bonds). The critical stretch of the bonds between the fibres is the same as that of the bonds within a fibre. Note that some fibres that appear to be crossing from the top-view in the $x y$-plane may actually not cross due to the depth (of $5 \mathrm{~nm}$ in the $z$-direction) of the nanofibre architecture used.

(B) Partially crosslinked, low variability in fibre-fibre bonds critical strain. Only $30 \%$ of the crossovers are bonded. These bonded crossovers are randomly selected from the total number of crossovers. The critical bond strain of the bonded crossovers is varied such that its distribution follows a random normal distribution with mean equal to the critical strain of bonds within a fibre. A standard deviation equal to $10 \%$ of the mean value (the mean value is 1 and the standard deviation we select is 0.1 ) is used.

(C) Partially crosslinked, high variability in fibre-fibre bonds critical strain. Same as case B, but with a standard deviation of 0.9 . We use the same 'map' of connected bonds as in case 

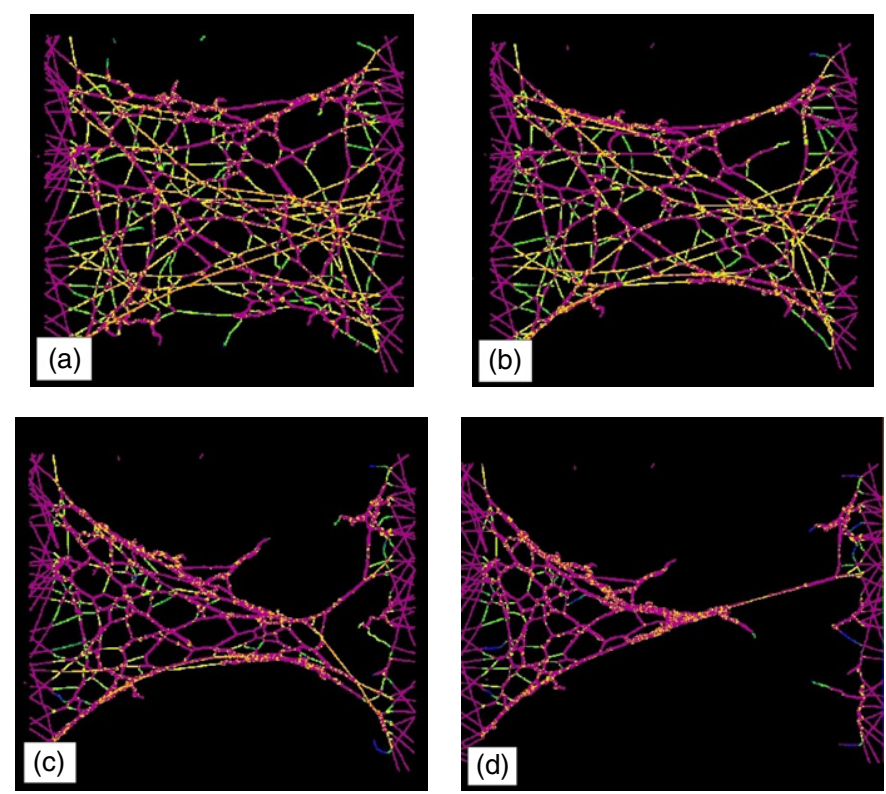

Figure 9. Uniaxial deformation of the nanofibre network showing the Poisson effect (vertical shrinking under horizontal stretching) due to the presence of van der Waals forces. The graphs are captured at (a) $4 \mathrm{~ns}$ or $7 \%$ strain, (b) $8 \mathrm{~ns}$ or $14 \%$ strain, (c) $14 \mathrm{~ns}$ or $25 \%$ strain and (d) $28 \mathrm{~ns}$ or $49.5 \%$ strain.

B; however, the critical bond strain associated with bonded crossovers will differ from those in case B.

In the partially crosslinked cases, some of the bonded crossovers break at strains lower than those of bonds along the fibre, while others break at strains larger than those of bonds along a fibre. We call the 'weaker' bonds 'sacrificial bonds'. The left and right ends of the network are moved away from one another with a constant velocity of $3 \mathrm{~nm} \mathrm{~ns}^{-1}$. The initial velocity profile along the network varies linearly from one end to the other, as in section 4 . We follow the deformation and damage of the network until total failure. In figure 10 we show the deformed configurations for the three cases at the same time stamp: 14 ns or $25 \%$ horizontal strain. The total force in the direction of motion ( $x$-axis) across the plane $x=0$ which the right half of the network exerts on the left half is plotted versus time in figure 11.

We observe that the peak force increases from case A to case B to case C. This is due, but only partly, to the fact that there are some bonds between fibres that have a larger critical stretch than the constant value of the critical stretch for the bonds within each fibre. Moreover, with the larger population of weaker crossovers in case $\mathrm{C}$ than in case $\mathrm{B}$, the network is able to rearrange itself more easily (by breaking the weak bonds first) and provide better strength in doing so due to the added accretion effects of the van der Waals interaction between different fibres. This is illustrated in figure 10, in which the saw-tooth behaviour of the load in case $\mathrm{C}$ is most pronounced since here is where we have the largest number of sacrificial bonds at the crossovers between fibres. The sacrificial bonds are broken, the network rearranges and a second force peak is reached. Case $\mathrm{C}$ also presents increased toughness, as well as increased peak load, because the additional saw-tooth peaks in figure 11 add to the area under the load-stretch curve, which is a measure of the work required to fail the network. 

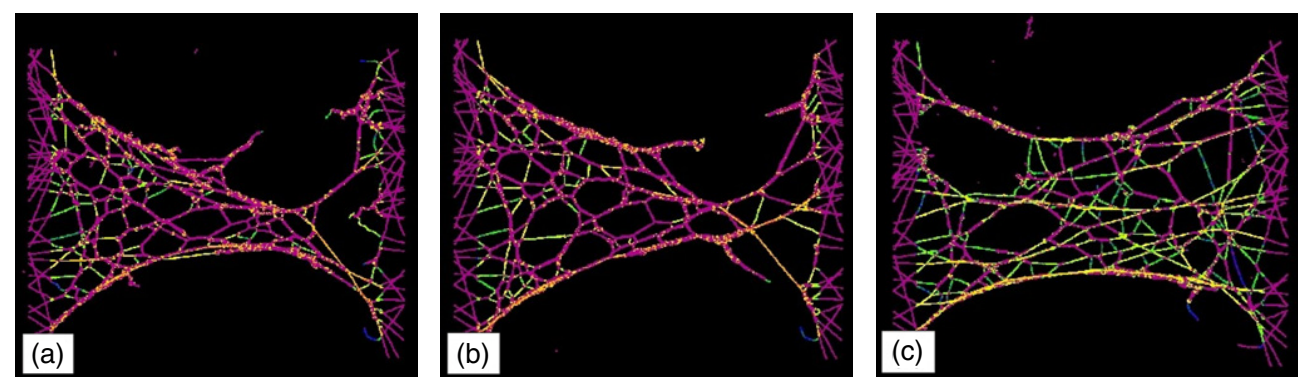

Figure 10. Deformed and damaged network when van der Waals forces are not present. $(a),(b)$ and $(c)$ figures correspond to the 'fully' bonded network, $30 \%$ bonded with standard deviation for the crosslink bonds strength of 0.1 and 0.9 , respectively. The plots are captured at $25 \%$ horizontal strain (or at $14 \mathrm{~ns}$ ).
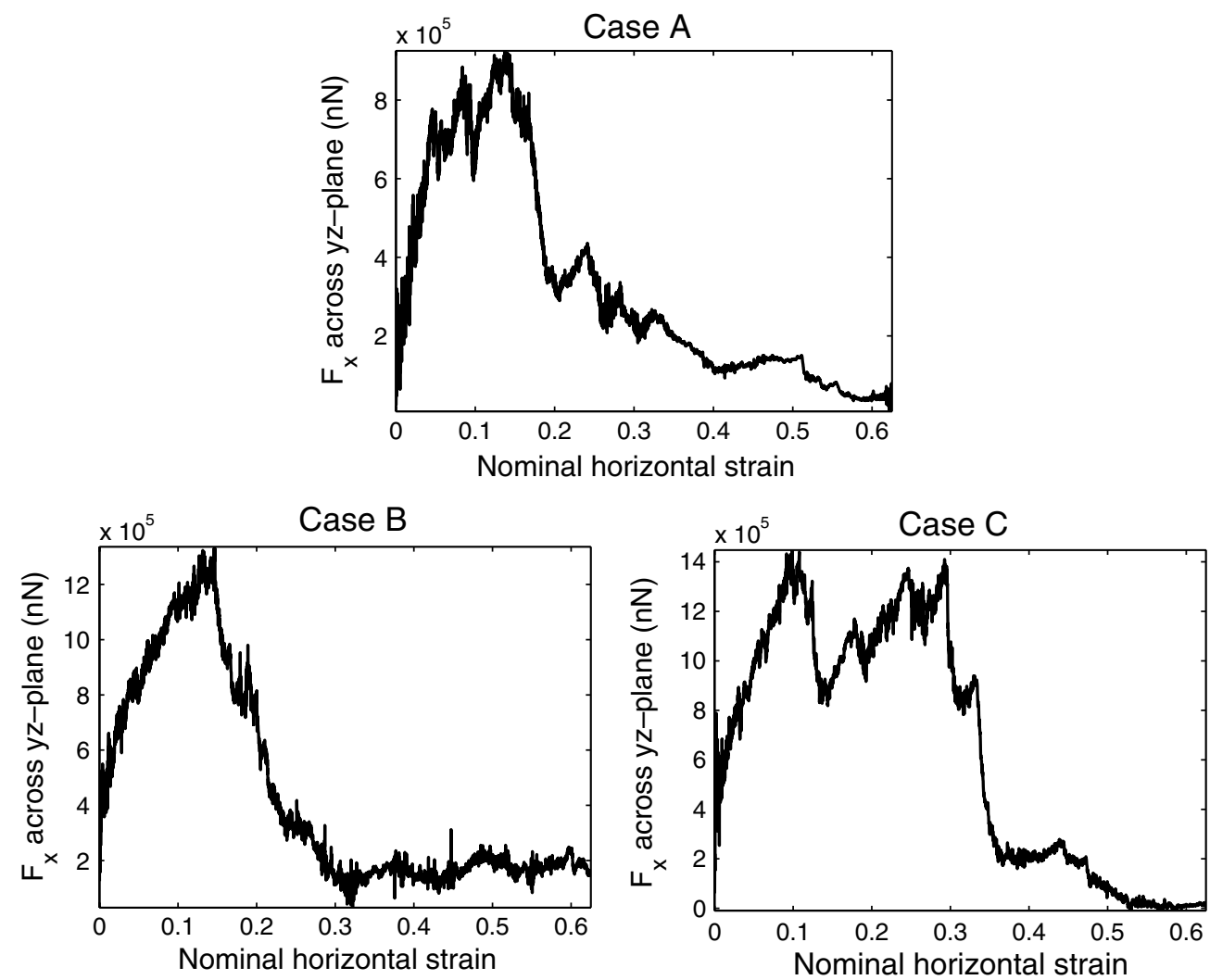

Figure 11. Time history of the $x$ component of the total force across the cutting plane $x=0$ that one half of the network acts on the other half.

The predicted enhancement in overall network toughness due to variability in the crossover strength appears to have a precedent in biology. While our system has a different configuration, we notice the similarity between the behaviour when sacrificial bonds are present and the experimental results observed when a natural polymer adhesive binding the microstructure of abalone shell is subjected to stretching in an AFM (see [41]). In this work [41] it was found that 

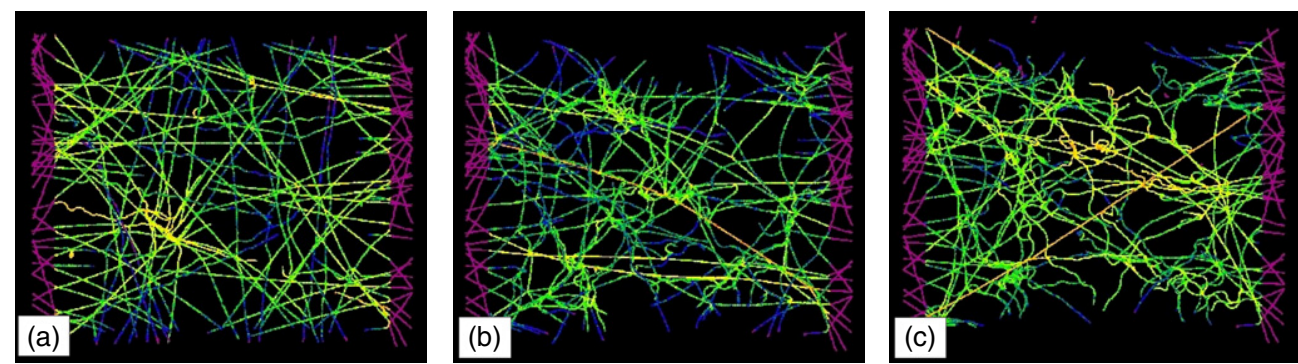

Figure 12. Deformed and damaged network when van der Waals forces are not present. $(a),(b)$ and $(c)$ figures correspond to the 'fully' bonded network, $30 \%$ bonded with standard deviations of 0.1 and 0.9 , respectively. The plots are at $25 \%$ horizontal strain (or $14 \mathrm{~ns}$ ).

the adhesive fibres elongate in a stepwise manner as folded domains or loops are pulled open. The elongation events occur for forces of a few hundred pNs, which are smaller than the forces of over $1 \mathrm{nN}$ required to break the polymer backbone in the threads. Smith et al [41] indicate that this 'modular' elongation mechanism might prove to be responsible for the extreme fracture toughness of the abalone shell. Ji and Gao [43] utilized the term 'sacrificial bonds' to explain the strength of natural protein-mineral nanocomposites. The protein modules deform between mineral platelets in the biological nanostructure and the force-extension relation for the protein is controlled by calcium ions linking negatively charged functional groups along the peptide chain. This results in protein unfolding instead of fracturing the molecular backbone [43].

If the van der Waals forces are not present, the nanofibre material system loses the nanoscale effects and behaves as a scale-free (macroscale) network of fibres. Using the same mapping of bonded contacts as in the case with van der Waals forces, as well as the same realization of the stochastic variations for the strength of the fibre-fibre bonds, we perform the corresponding numerical tests as above, the only difference being the absence of van der Waals forces. The results with the deformed network at $14 \mathrm{~ns}$ and the force-time history are presented in figures 12 and 13, respectively. The increase in strength from case A to case B occurs here as well, since together with sacrificial bonds we also introduce stronger bonds. However, in contrast to the situation when van der Waals forces are present, there is a decrease in strength from case B to case C. Inertial effects are also more pronounced for the case with larger heterogeneity than for the other cases.

\section{Conclusions}

We have analysed the behaviour of nanofibre networks under dynamic deformation. For this we utilized the peridynamic method to capture fracture and damage of the individual nanofibres and the network. Our models included van der Waals forces between different fibres in addition to microelastic peridynamic forces acting along the fibres and between the fibres at crosslinks. The PF allowed for a natural consideration of the van der Waals interaction alongside the entropic elastic forces.

Biaxial computational simulations showed the formation of cellular pattern damage, observed in other cases of thin-membrane damage. The cellular damage appeared only when van der Waals interactions were included in the simulations. Introduction of additional heterogeneity in the strength of crosslink bonds between different fibres showed that 'weaker' (a network with fewer crosslinks and with a larger spread of bond strengths) may mean 'stronger' when van der Waals forces act. The force response across the nanofibre network was noted to 

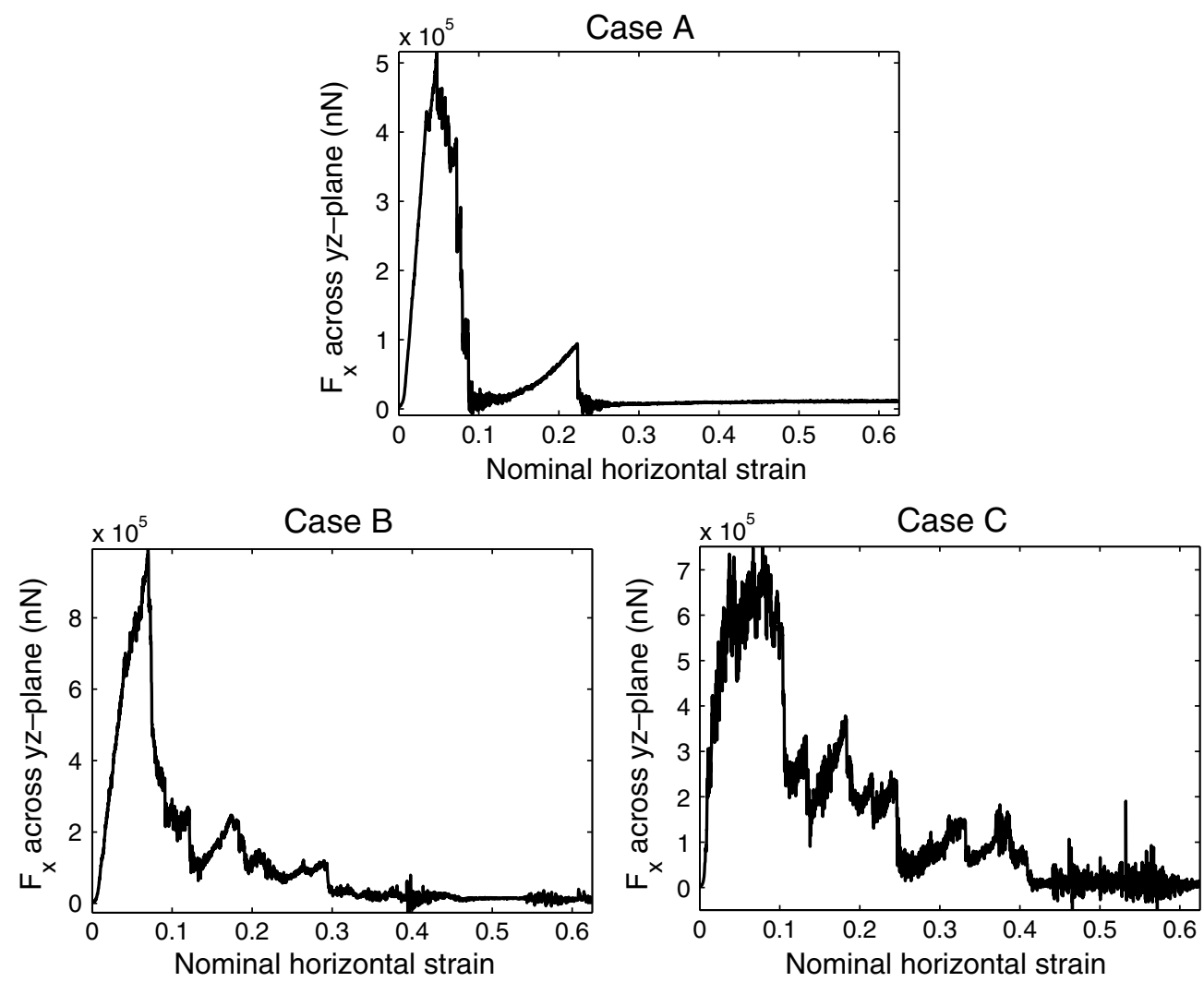

Figure 13. Time history of the $x$ component of the total force across the cutting plane $x=0$ that one half of the network acts on the other half.

present similar features with force profiles observed experimentally in the rupturing of protein adhesive in abalone shells.

This study led to the following observations with implications in the analysis and design of nanostructured materials:

- van der Waals interactions are important in increasing the strength and toughness of the nanofibre network;

- heterogeneity in the strength of bonds in the nanofibre network (the existence of sacrificial bonds) can increase its fracture energy dissipation/toughness/ductility (saw-tooth force profiles);

- reorientation due to breakage and deformation, and a Poisson effect due to van der Waalsinduced accretion, are the two main mechanisms that control the dynamic deformation and damage of nanofibre networks.

\section{Acknowledgments}

This work was supported in part by the Computer Science Research Institute at Sandia National Laboratories, Albuquerque, New Mexico, through a summer research fellow grant and by the US Army Research Laboratory under the RMAC-RTP Cooperative Agreement No W911NF-04-2-0011. The author is extremely grateful to Dr Stewart A Silling from Sandia 
for continued guidance and support and numerous discussions. The author also thanks Drs Abe Askari, Etienne Emmrich, Olaf Weckner, Jifeng Xu and Mijia Yang for useful discussions. This work was completed utilizing the Research Computing Facility of the University of Nebraska-Lincoln. Han Jiang helped run some of the numerical tests.

\section{References}

[1] Li D and Xia Y 2004 Electrospinning of nanofibers: reinventing the wheel? Adv. Mater. 16 1151-70

[2] Dzenis Y 2004 Spinning continuous fibers for nanotechnology Science 304 1917-19

[3] Ramakrishna S, Fujihara K, Teo W-E, Lim T-C and Ma Z 2005 An Introduction to Electrospinning of Nanofibers (Singapore: World Scientific)

[4] Stevens M M and George J H 2005 Exploring and engineering the cell surface interface Science 310 $1135-8$

[5] Reneker D H, Yarin A L, Fong H and Koombhongse S 2000 Bending instability of electrically charged liquid jets of polymer solutions in electrospinning J. Appl. Phys. 87 4531-47

[6] Yarin A L, Koombhongse S and Reneker D H 2001 Taylor cone and jetting from liquid droplets in electrospinning of nanofibers J. Appl. Phys. 90 4836-46

[7] Bobaru F and Silling S A 2004 Peridynamic 3d models of nanofiber networks and carbon nanotube-reinforced composites Materials Processing and Design: Modeling, Simulation and Applications NUMIFORM (Columbus, Ohio, 2004) ed S Ghosh et al (New York: AIP) pp 1565-70

[8] Bobaru F, Silling S A and Jiang H 2005 Peridynamic fracture and damage modeling of membranes and nanofiber networks The 11th Int. Conf. on Fracture (Turin, Italy, 1-6 2005) paper no. 5748 pp 1-6

[9] Sastry A M, Cheng X and Wang C W 1998 Mechanics of stochastic fibrous networks J. Thermoplast. Compos. Mater. 11 288-96

[10] Wang C W, Berhan L and Sastry A M 2000 Structure, mechanics and failure of stochastic fibrous networks: I. Microscale considerations J. Eng. Mater. Technol., Trans. ASME 122 450-9

[11] Wang C W and Sastry A M 2000 Structure, mechanics and failure of stochastic fibrous networks: II. Network simulations and application J. Eng. Mater. Technol., Trans. ASME 122 460-8

[12] Berhan L and Sastry A M 2003 On modeling bonds in fused, porous networks: 3d simulations of fibrousparticulate joints J. Compos. Mater. 37 715-40

[13] Yi Y B, Berhan L and Sastry A M 2004 Statistical geometry of random fibrous networks, revisited: waviness, dimensionality, and percolation J. Appl. Phys. 96 1318-27

[14] Astrom J A, Makinen J P, Alava M J and Timonen J 2000 Elasticity of poissonian fiber networks Phys. Rev. E $615550-6$

[15] Astrom J A, Makinen J P, Hirvonen H and Timonen J 2000 Stiffness of compressed fiber mats J. Appl. Phys. 88 5056-61

[16] Wu X-F and Dzenis Y A 2005 Elasticity of planar fiber networks J. Appl. Phys. 98 093501-9

[17] Zohdi T I and Steigmann D J 2002 The toughening effect of microscopic filament misalignment on macroscopic ballistic fabric response Int. J. Fract. 118 L71-6

[18] Gonzalez C and Llorca J 2003 A numerical model to simulate the deformation and fracture of polyethylene fibres Modelling Simul. Mater. Sci. Eng. 11 349-64

[19] Israelachvili J 1992 Intermolecular and Surface Forces 2nd edn (NewYork: Academic)

[20] Gao H and Yao H 2004 Shape insensitive optimal adhesion of nanoscale fibrillar structures Proc. Natl Acad. Sci. 101 7851-6

[21] Silling S A 2000 Reformulation of elasticity theory for discontinuities and long-range forces J. Mech. Phys. Solids 48 175-209

[22] Eringen A C and Edelen D G B 1972 On non-local elasticity Int. J. Eng. Sci. 10 233-48

[23] Eringen A C 1987 Theory of nonlocal elasticity: some applications Res. Mech. 21 313-42

[24] Kunin I A 1982 Elastic Media with Microstructure I: One-Dimensional Models (Springer Series in Solid-State Sciences) (Berlin: Springer)

[25] Silling S A, Zimmermann M and Abeyaratne R 2003 Deformation of a peridynamic bar J. Elast. 73 173-90

[26] Silling S A and Bobaru F 2005 Peridynamic modeling of membranes and fibers Int. J. Non-Lin. Mech. 40 395-409

[27] Silling S A and Askari E 2005 A meshfree method based on the peridynamic model of solid mechanics Comput. Struct. 83 1526-35

[28] Bazant Z P and Jirasek M 2002 Nonlocal integral formulations of plasticity and damage: survey of progress J. Eng. Mech. 128 1119-49

[29] Griffiths D V and Mustoe G G W 2001 Modelling of elastic continua using a grillage of structural elements based on discrete element concepts Int. J. Numer. Methods Eng. 50 1759-75 
[30] Cusatis G, Bazant Z P and Cedolin L 2003 Confinement-shear lattice model for concrete damage in tension and compression: I. Theory J. Eng. Mech. 129 1439-48

[31] Cusatis G, Bazant Z P and Cedolin L 2003 Confinement-shear lattice model for concrete damage in tension and compression: I. Computation and validation J. Eng. Mech. 129 1449-58

[32] Cusatis G, Bazant Z P and Cedolin L 2003 Confinement-shear lattice csl model for fracture propagation in concrete Comput. Methods Appl. Mech. Eng. 195 7154-71

[33] Weckner O and Abeyaratne R 2005 The effect of long-range forces on the dynamics of a bar J. Mech. Phys. Solids $\mathbf{5 3} 705-28$

[34] Bobaru F and Silling S A 2006 Peridynamic fracture of brittle polycrystalline ceramics 7th World Congress on Computational Mechanics (Los Angeles, California Jul. 2006)

[35] Zhou Y, Freitag M, Hone J, Staii C and Johnson A T 2003 Fabrication and electrical characterization of polyaniline-based nanofibers with diameter below $30 \mathrm{~nm}$ Appl. Phys. Lett. Issue 83 3800-2

[36] Xie W 2005 Peridynamic flux-corrected transport algorithm for shock wave studies Master's Thesis University of Nebraska-Lincoln, Lincoln, Nebraska

[37] Bobaru F, Yang M, Alves L F, Silling S A and Askari A 2007 Adaptive refinement, scaling, and convergence in peridynamics: the 1-dimensional case (to be submitted)

[38] Bobaru F, Xie W and Yang M 2007 Impact and spallation in brittle solids using peridynamics 17th U.S. Army symposium on Solid Mechanics (Baltimore, Maryland Apr. 2007) pp 205-6

[39] Zussman E, Burman M, Yarin A L, Khalfin R and Cohen Y 2006 Tensile deformation of electrospun nylon-6,6 nanofibers J. Polym. Sci. B: Polym. Phys. 44 1482-9

[40] Silling S A 2001-2005 Emu: A Parallel Implementation of Peridynamics Sandia National Laboratories

[41] Smith B L et al 1999 Molecular mechanistic origin of the toughness of natural adhesives, fibres and composites Nature 399 761-3

[42] Thompson J B, Kindt J H, Drake B, Hansma H G, Morse D E and Hansma P K 2001 Bone indentation recovery time correlates with bond reforming time Nature 414 773-6

[43] Ji B and Gao H 2004 Mechanical properties of nanostructure of biological materials J. Mech. Phys. Solids 52 1963-90 